\title{
Aspects of design antennas for radio occultation method of ionosphere diagnostics
}

\author{
Alexander Generalov ${ }^{1, *}$,Elchin Gadzhiev ${ }^{1}$, Pavel Shmachilin ${ }^{2}$, Yuri Polushkovskiy ${ }^{3}$, \\ Vladimir Skripachev ${ }^{3}$, and Mihael Tumanov ${ }^{3}$ \\ ${ }^{1}$ JSC "Research Institute for Electromechanics",143502 st. Panfilov 11, Russia \\ ${ }^{2}$ Moscow Aviation Institute (National Research University) "MAI", 125993 Volokolamskoe \\ Highway, 4, Russia \\ ${ }^{3}$ The Federal Center of Analyzis 109316 st. Talalikhina, 33, Russia
}

\begin{abstract}
The ionosphere is the ionized part of Earth's upper atmosphere, from about $60 \mathrm{~km}$ to $1,000 \mathrm{~km}$ altitude, a region that includes the thermosphere and parts of the mesosphere and exosphere. The ionosphere is ionized by solar radiation. It plays an important role in atmospheric electricity and forms the inner edge of the magnetosphere. It has practical importance because, among other functions, it influences radio propagation to distant places on the Earth. The region below the ionosphere is called neutral atmosphere, or neutrosphere. In this paper aspects of design antennas for radio occultation method of ionosphere diagnostics are presented.
\end{abstract}

\section{Introduction}

Ionosphere as a medium of propagation, affects the characteristics of the radio signal-amplitude, frequency, phase, group delay and polarization of radio waves, which in turn has a significant impact on the quality of communication "spacecraft-spacecraft", "spacecraft-Earth", as well as the accuracy of navigation definitions on signals from navigation satellites.

Ionospheric effects are used not only to study ionospheric plasma, but also to calculate the necessary corrections in order to improve the accuracy of navigation determination.

Thus, the diagnosis of the state of the ionosphere is an actual, modern task.

In this paper to improve the efficiency of sensing on small and large spacecraft through the use of simple but more efficient antennas compared to those currently used is proposed.

\section{Perspective of development}

Global Navigation Satellite System (GNSS) receiver mounted on a low Earth orbit (LEO) satellite is useful for making a radio occultation. The GNSS radio signals transmitted from GNSS transmitters to LEO satellite's receiver are bent when the radio

* Corresponding author: gadzhiev_elchin@mail.ru 
signal goes through atmosphere and ionosphere of Earth. Because GNSS and LEO satellites are moving at the same time, the radio signal path sounding the atmosphere and ionosphere.

The radio occultation divided by two types [1]:

- $\quad$ atmospheric occultation (lower than $90 \mathrm{~km}$ );

- ionospheric occultation (from $90 \mathrm{~km}$ to LEO altitude).

The schema of radio occultation is shown on the Fig. 1:

G1 - occultation GNSS satellite,

G2 - reference GNSS satellite,

L - LEO satellite with onboard GNSS receiver,

Gn ... Gn+2 - other GNSS satellites for solving primary navigation task for LEO satellite,

A - ground station for processing data,

$\mathrm{T}$ - bending point of radio signal.

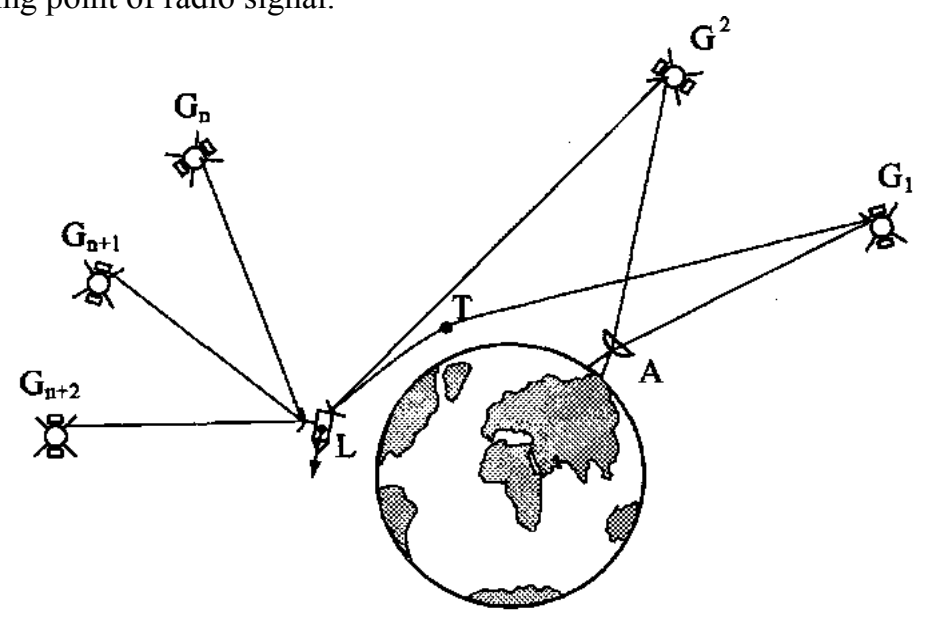

Fig 1. The schema of radio occultation method.

The main sources of errors in radio occultation method are [2]:

- $\quad$ radio signal to noise performance;

- clock error;

- multipath propagation;

- orbit determination error;

- retrieval error et al.

To minimizes errors and get well quality of occultation information is necessary developed special antennas.

\section{Antenna system}

It is known that a multibeam onboard antenna with a high gain is required, which can receive reference signals from ten or more navigation satellites, as well as register their corresponding reflections from the vicinity of mirror points on the earth's surface.

It is proposed to improve the efficiency of sensing on small spacecraft through the use of simple but more effective antennas compared to those currently used [3].

In the course of clarifying the requirements for the antenna, the following characteristics were obtained from the developers of the sensing equipment:

- a gain of approximately $10 \mathrm{~dB}$;

- $\quad$ radiation pattern in azimuth $60^{\circ}$ to $90^{\circ}$; 
- $\quad$ radiation pattern in elevation from $20^{\circ}$ to $30^{\circ}$;

- $\quad$ working frequency: $1.6 \mathrm{GHz}$ or $1.2 \mathrm{GHz}$.

It is possible to use antennas with uneven radiation pattern.

Further, the following options were considered to solve the problem of constructing antennas for sensing equipment [4]:

- $\quad$ spiral emitter (the number of turns of the spiral 8);

- $\quad$ spiral emitter (the number of spiral turns 11);

- helical antenna array of four elements (the number of turns of the spiral 6);

- helical antenna array of four elements (the number of turns of the spiral 8);

- antenna array of two elements, for example, microstrip radiators;

- $\quad$ microstrip (printed) emitter;

- horn radiator with a helical exciter.

For example, variant of design of on-board antenna is shown at Fig.2

Fig 2. The on-board helical antenna array.

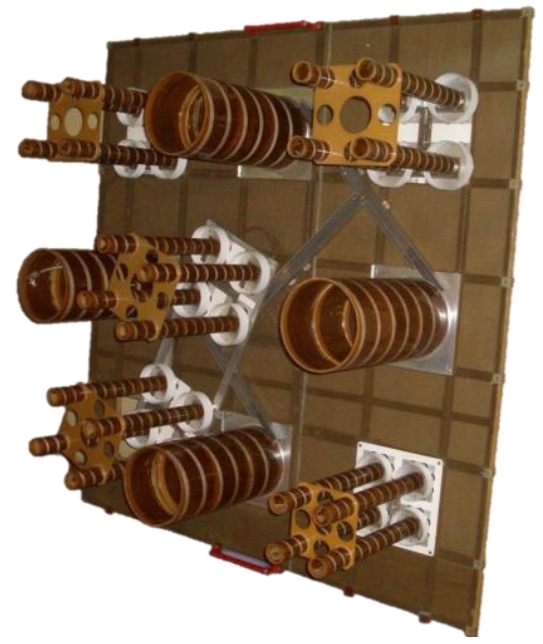

The on-board helical antenna array consists from different types of helical emitters as shown at Fig. 2. Each helical emitter can be used as on-board antenna as shown at Fig. 3.

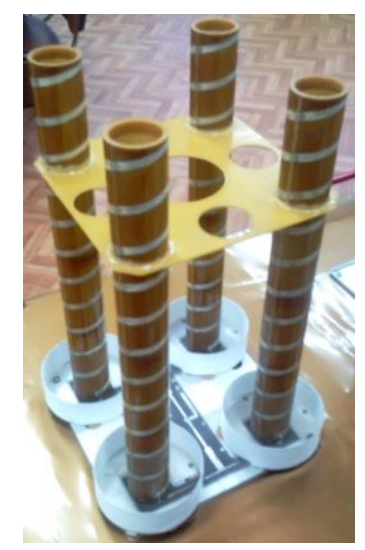

Fig 3. The on-board helical antenna array.

Also in this paper the main characteristics of presented on-board antennas such as voltage standing-wave ratio, radiation pattern, gain, axial ratio and etc are presented. The obtained results satisfy the specified requirements. 


\section{Conclusion}

The diagnosis of the state of the ionosphere is an actual, modern task. The use of the onboard antennas considered in this paper will improve the performance scientific experiments on sounding the atmosphere, the ionosphere and the earth's surface from space using spacecraft, including small, and the proposed options for the construction of antennas of this equipment will allow developers to choose the best option depending on the spacecraft on which the sensing equipment will be installed.

\section{References}

1. Shuanggen Jin, Estel Cardellach, Feiqin Xie. GNSS Remote Sensing: Theory, Methods and Applications / Springer, 276 p. (2014)

2. Kursinski ER, Hajj GA, Schofield JT, Linfield RP, Hardy KR (1997) Observing Earth's atmosphere with radio occultation measurements using the Global Positioning System. J Geophys Res 102(D19):23429-23465

3. V.S. Bocharov, A.G. Generalov, E.V. Gadzhiev. Variants of spacecraft antenna design for space surface sensing, Antenny, 4 (215), pp. 3-8 (2015)

4. A.G. Generalov, E.V. Gadzhiev, M.R. Salikhov. Spiral antennas application for prospective onboard systems and complexes, Trudy MAI, 106, (2019) http://trudymai.ru/eng/published.php?ID=105685\&eng=Y 